\title{
Water trimer torsional spectrum from accurate $a b$ initio and semiempirical potentials
}

\author{
Ad van der Avoird ${ }^{a)}$ \\ Theoretical Chemistry, Institute for Molecules and Materials, Radboud University Nijmegen, \\ Toernooiveld 1, 6525 ED Nijmegen, The Netherlands
}

Krzysztof Szalewicz ${ }^{\text {) }}$

Department of Physics and Astronomy, University of Delaware, Newark, Delaware 19716, USA

(Received 27 September 2007; accepted 22 October 2007; published online 3 January 2008)

\begin{abstract}
The torsional levels of $\left(\mathrm{H}_{2} \mathrm{O}\right)_{3}$ and $\left(\mathrm{D}_{2} \mathrm{O}\right)_{3}$ were calculated in a restricted dimensionality (three-dimensional) model with several recently proposed water potentials. Comparison with the experimental data provides a critical test, not only of the pair interactions that have already been probed on the water dimer spectra, but also of the nonadditive three-body contributions to the potential. The purely ab initio CC-pol and HBB potentials that were previously shown to yield very accurate water dimer levels, also reproduce the trimer levels well when supplemented with an appropriate three-body interaction potential. The TTM2.1 potential gives considerably less good agreement with experiment. Also the semiempirical VRT(ASP-W)III potential, fitted to the water dimer vibration-rotation-tunneling levels, gives substantial disagreement with the measured water trimer levels, which shows that the latter probe the potential for geometries other than those probed by the dimer spectrum. Although the three-body nonadditive interactions significantly increase the stability of the water trimer, their effect on the torsional energy barriers and vibration-tunneling frequencies is less significant. () 2008 American Institute of Physics. [DOI: 10.1063/1.2812556]
\end{abstract}

\section{INTRODUCTION}

The water trimer was first characterized experimentally in 1992 by means of terahertz spectroscopy. ${ }^{1}$ Since then, a number of torsional bands of $\left(\mathrm{H}_{2} \mathrm{O}\right)_{3}$ and $\left(\mathrm{D}_{2} \mathrm{O}\right)_{3}$ have been added $^{2-5}$ to the first observation, which completely determine the torsional levels of these two isotopomers up to energies of about 150 and $100 \mathrm{~cm}^{-1}$, respectively. Many calculations have been performed on $\left(\mathrm{H}_{2} \mathrm{O}\right)_{3},\left(\mathrm{D}_{2} \mathrm{O}\right)_{3}$, and the mixed isotope forms of the water trimer in order to theoretically describe this manifold of states. ${ }^{4-24}$ In most cases semiempirical potentials were used in these calculations or, when $a b$ initio potentials were applied, they were specifically calculated for the restricted dimensionality model used in the computation of the torsional levels. In a few of the papers $^{6,14,21}$ the trimer levels of $\left(\mathrm{H}_{2} \mathrm{O}\right)_{3}$ and $\left(\mathrm{D}_{2} \mathrm{O}\right)_{3}$ were used to test a global $a b$ initio water force field including pair and three-body interactions. Reference 25 reviews the work up to the year 2000. Several ab initio water pair potentials have been obtained recently, with ${ }^{26-29}$ or without ${ }^{30,31}$ explicit inclusion of the polarization effects that form the dominant contribution to the many-body interactions in water. These water pair potentials were tested by a computation ${ }^{29,31}$ of the vibration-rotation-tunneling (VRT) levels of the water dimer and comparison with the available high-resolution terahertz spectra. ${ }^{32-40}$ The same spectra were used to obtain semiempirical water potentials, ${ }^{41-43}$ dubbed VRT(ASP-W), by starting from an $a b$ initio potential and reoptimizing some of the parameters in the analytic representation of the potential

\footnotetext{
${ }^{a)}$ Electronic mail: avda@theochem.ru.nl.

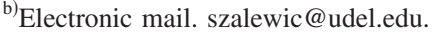

surface by a fit of computed water dimer levels to the available experimental data. ${ }^{37-40}$ The ab initio CC-pol potential of Refs. 27-29 and the ab initio HBB potential of Ref. 31 turned out to reproduce the spectra of $\left(\mathrm{H}_{2} \mathrm{O}\right)_{2}$ and $\left(\mathrm{D}_{2} \mathrm{O}\right)_{2}$ very precisely, even more so than the best of the VRT(ASP-W) potentials ${ }^{42}$ fitted to the latter spectrum. Several of these potentials ${ }^{26-29,42,44}$ have also been used in molecular dynamics (MD) simulations of liquid water. An extensive discussion of their performance in this respect can be found in Ref. 29. The SAPT-5st potential ${ }^{6}$ which was obtained by an empirical tuning of the SAPT- 5 s potential ${ }^{44}$ to only a single experimental value, remains overall the best potential in predicting the spectra of the dimer, but this potential gave less satisfactory results in the simulations of liquid water.

The present paper describes our calculations of the water trimer torsional levels from the potentials discussed above, with the inclusion of the three-body polarization interactions when the potential accounts for those. ${ }^{26-28,42}$ With the CC-pol potential of Refs. 27 and 28 three-body exchange interactions are available as well, ${ }^{45}$ and we included these in the calculation of the water trimer levels. The HBB potential of Refs. 30 and 31 is a pure pair potential, so we supplemented it with the three-body polarization and exchange contributions from Ref. 45. The comparison of the computed water trimer levels with the measured data provides a test of the complex three-body contributions to the water force field and, as it can be seen below, it also provides a critical test of the different water pair potentials for geometries that are not probed by the dimer spectra. 


\section{THEORY}

The minimum energy structure of the water trimer, an asymmetric ring (symmetry group $C_{1}$ ) held together by three hydrogen bonds, has been established by many $a b$ initio calculations. ${ }^{45-49}$ Two of the non-hydrogen-bonded ("free") hydrogen atoms are on the same side of the oxygen plane, the third one is on the opposite side. Experimentally it has been demonstrated that this nonplanar asymmetric structure vibrationally averages to a planar symmetric top via the torsional (flipping) motion. ${ }^{1,2}$ The torsional quantum levels of the water trimer have been considered at various levels of theory. The first, and simplest, was a one-dimensional treatment by Schütz et al. who used just a cosine function as the potential. ${ }^{23,24}$ Their calculation obtained the correct ordering of the energy levels but gave poor quantitative results. Model torsional (three-dimensional) potential energy surfaces fitted to $a b$ initio calculated points were created by Bürgi et al. ${ }^{16}$ and by van Duijneveldt-van de Rijdt and van Duijneveldt. ${ }^{20}$ Several dynamics calculations have been performed on these potentials including two-dimensional ${ }^{18}$ and three-dimensional ${ }^{12,13,19}$ treatments. The three-dimensional calculations by van der Avoird and co-workers ${ }^{12,13}$ include the overall rotation of the trimer complex and the Coriolis coupling to the torsional motion. They also led to an effective rotation-tunneling Hamiltonian that was used successfully to interpret and extract physically meaningful parameters from the measured high-resolution spectra. ${ }^{4,5} \mathrm{~A}$ fourdimensional $a b$ initio potential involving the torsional degrees of freedom and the symmetric hydrogen-bond stretch mode was computed by Sabo et al.,${ }^{8-10}$ who used this potential in a four-dimensional dynamics calculation and investigated the effect of torsional and stretch excitations on the rotational constants of $\left(\mathrm{H}_{2} \mathrm{O}\right)_{3}$ and $\left(\mathrm{D}_{2} \mathrm{O}\right)_{3}$.

The second type of internal large amplitude motion in the water trimer is bifurcation tunneling (also called donor tunneling). This is a rearrangement process in which a single water monomer exchanges its hydrogen-bonded and free hydrogen atoms by tunneling through a bifurcated transition state. It could be observed experimentally ${ }^{2}$ and unambiguously identified, since it gives rise to a splitting of the torsional levels and transitions into quartets. The splitting between the lines in these quartets, typically about $300 \mathrm{MHz}$ for $\left(\mathrm{H}_{2} \mathrm{O}\right)_{3}$ and about $5 \mathrm{MHz}$ for $\left(\mathrm{D}_{2} \mathrm{O}\right)_{3}$, is much smaller than the energy gaps between the torsional levels. This is because the corresponding barrier height, calculated ${ }^{45,46,50,51}$ to be about $750 \mathrm{~cm}^{-1}$, is substantially higher than the torsional barrier. Quantitative calculations of the level splittings produced by this tunneling motion would require 12dimensional quantum calculations of the coupled intermolecular motions of the trimer. To our knowledge, such calculations have only been done in the semiclassical WentzelKramers-Brillouin (WKB) approximation. ${ }^{52}$

The construction of the torsional manifold for the water trimer, including a group theoretical treatment, has been detailed in several previous papers. ${ }^{2,12,13,22,53,54}$ Also the bifurcation tunneling splitting of the levels was considered in some of these references, but only qualitatively. Before we describe the calculation of the torsional levels on different

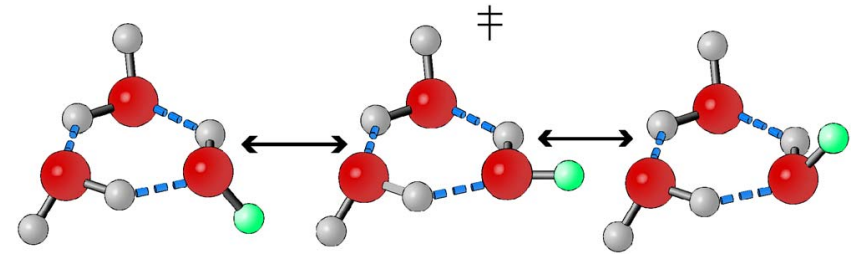

FIG. 1. (Color online) Torsional flipping in the water trimer between the dud and uud equilibrium structures.

potential surfaces, let us briefly review the basic theory behind the torsional tunneling dynamics in the water trimer.

The equilibrium structure of the water trimer may interconvert between six of the 96 equivalent minima covering the 12-dimensional intermolecular potential energy surface through a simple, low barrier flip of a free hydrogen from one side of the oxygen framework to the other. If we denote the free hydrogens above the plane of the oxygens by "up" or " $u$ " and those below by "down" or " $d$," we may say that a structure $u u d$ flips to an equivalent structure $d u d$, see Fig. 1. Further consecutive flips produce the $d u u, d d u, u d u$, and $u d d$ structures. With another additional flip, the system returns to the original uud structure. So we see that starting from one of these six equilibrium structures one ultimately visits six equivalent minima through a cyclic process of singlehydrogen $u$ - $d$ flips. Since the energy barrier that has to be overcome by a single flip is rather low, about $80 \mathrm{~cm}^{-1},{ }^{20,45,46,50,51,55}$ this rearrangement mechanism gives rise to large tunneling splittings.

The permutation-inversion (PI) symmetry group associated with the torsional flipping between the six equivalent minima on the potential surface is the cyclic group $G_{6}$, isomorphic to the point group $C_{3 h}$. The complex irreducible representations (irreps) of this group are conveniently labeled by the quantum number $k=0, \pm 1, \pm 2,3$. A diagram showing the six lowest energy torsional vibrational levels of $\left(\mathrm{H}_{2} \mathrm{O}\right)_{3}$, along with the observed transitions, is given in Fig. 2. The symmetry of these levels has been compared $^{22}$ to a

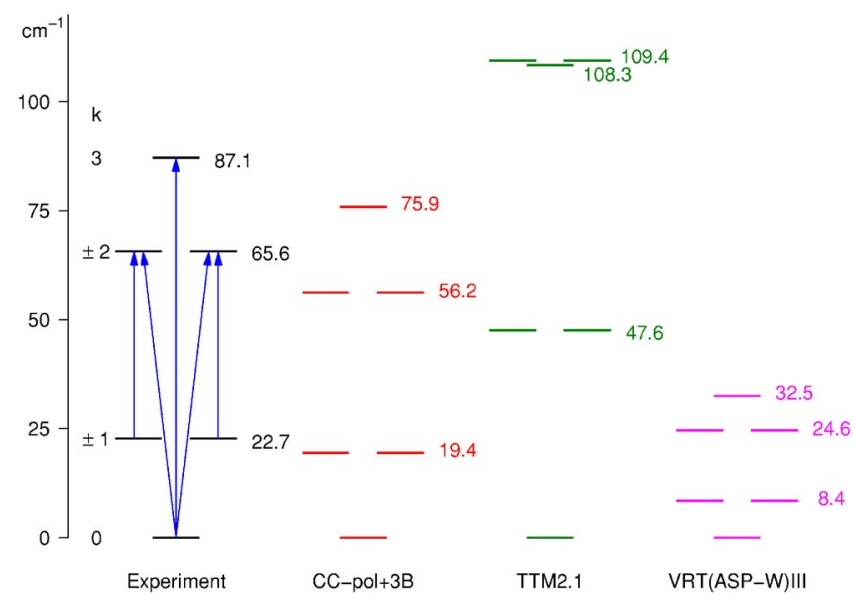

FIG. 2. (Color online) Torsional levels $\left(\mathrm{in}^{-1}\right)$ of $\left(\mathrm{H}_{2} \mathrm{O}\right)_{3}$ from experiment (Ref. 4) and from calculations with different potentials. The label $k$ $=0, \pm 1, \pm 2,3$ corresponds to the irreducible representations of the cyclic permutation-inversion group $G_{6}$. Arrows indicate the observed transitions. The levels from the $\mathrm{HBB}+3 \mathrm{~B}$ potential are not shown, because these are very similar to the $\mathrm{CC}-$ pol $+3 \mathrm{~B}$ levels. 
Hückel treatment of the $\pi$ electron system of benzene. The levels with $k=0$ and $k=3$ are nondegenerate, as the $A$ and $B$ levels are in benzene, while those with $k= \pm 1$ and $k= \pm 2$ are twofold degenerate, similarly to the benzene $E$ levels. The energy increases with $|k|$, the number of nodes in the real wave functions (which for the degenerate levels are plus and minus combinations of the complex functions with $k= \pm 1$ and $k= \pm 2$ ). Transitions between torsional energy levels of symmetry $k$ and $k^{\prime}$ follow the selection rule,

$$
(k-K)-\left(k^{\prime}-K^{\prime}\right)=3(\text { modulo } 6),
$$

where $K$ is the projection of the total angular momentum $J$ of the trimer on the threefold symmetry axis. This rule allows the permitted transitions to be easily determined, as well as the band polarization (parallel or perpendicular). The $k=3 \leftarrow 0$ transition observed at $87.1 \mathrm{~cm}^{-1}$ in $\left(\mathrm{H}_{2} \mathrm{O}\right)_{3}$, for example, is only allowed for $\Delta K=K^{\prime}-K=0$, and hence is observed as a parallel band. The $65.6 \mathrm{~cm}^{-1}$ vibrational band is made up of two distinct perpendicular vibrational subbands, as $k=+2 \leftarrow 0$ is only allowed when $\Delta K=-1$, while $k=-2 \leftarrow 0$ is only allowed when $\Delta K=+1$, see Fig. 2 . The third band, observed at $42.9 \mathrm{~cm}^{-1}$, arises from the two parallel subbands $k=+2 \leftarrow-1$ and $k=-2 \leftarrow+1$. The degenerate subbands are severely perturbed by Coriolis effects, but with the effective Hamiltonian derived in Ref. 5, the fine structure in these bands could be very well interpreted and fitted. ${ }^{4,5}$

The bifurcation tunneling process, along with the flipping process discussed above, creates two distinct sets of 48 interconvertable equivalent minima on the water trimer potential energy surface and generates the molecular symmetry group for the water trimer, $G_{48}$. The group theory for these rearrangement processes has been examined extensively in a number of papers. ${ }^{2,12,22}$ The accompanying tunneling splittings are not amenable to accurate calculations yet, so from here on we will concentrate on the torsional motions and the accompanying flipping process.

\section{CALCULATIONS}

The restricted-dimensionality model and the Hamiltonian that we used to compute the torsional energy levels in the water trimer are described in detail in Ref. 12. In this model, the distances between the centers of mass of the monomers are frozen and the hydrogen bonded "framework" of the water trimer is kept rigid and planar. The free hydrogen atoms are allowed to move from above to below this plane by performing a torsional motion about the axis through the monomer center of mass and the bound hydrogen. The torsional angles of the monomers $A, B$, and $C$ are denoted by $\chi_{A}, \chi_{B}, \chi_{C}$. In order to maintain the threefold symmetry observed experimentally, the distances $R_{A B}=R_{B C}$ $=R_{A C}=R$ between the three centers of mass were kept equal, and so were the three angles $\theta_{A}=\theta_{B}=\theta_{C}=\theta$ between the torsional axes of monomers $A, B$, and $C$ and the axes that connect the centers of mass. Moreover, the torsional axes were constrained to be in the plane through the centers of mass. For the rigid-monomer potentials CC-pol and VRT(ASP-W)III, the monomer $\mathrm{OH}$ bond length and $\mathrm{HOH}$ angle were taken for which these potentials are defined, while for the flexible-monomer potentials TTM2.1 and HBB we chose the free-monomer equilibrium values. The threebody potential of Ref. 45 is defined for the same $\mathrm{OH}$ bond length and $\mathrm{HOH}$ angle as the $\mathrm{CC}$-pol potential; these are the $\mathrm{H}_{2} \mathrm{O}$ ground state vibrationally averaged values. The centerof-mass distance $R$ and the angle $\theta$ were optimized for each potential used in the calculations by searching for the equilibrium geometry of $\left(\mathrm{H}_{2} \mathrm{O}\right)_{3}$ (with $R_{A B}=R_{B C}=R_{A C}=R$, and $\left.\theta_{A}=\theta_{B}=\theta_{C}=\theta\right)$. The framework was then kept rigid at the equilibrium geometry, so that an effective torsional potential $V\left(\chi_{A}, \chi_{B}, \chi_{C}\right)$ is obtained. We also determined the torsional barrier $\Delta E$, i.e., the barrier to a single flipping motion. In agreement with the literature ${ }^{20,46}$ we define this barrier by determining the energy of the upd structure, where $p$ (for "planar") indicates that one of the monomers has its free hydrogen atom in the plane of the hydrogen-bonded framework. We did this by starting from the equilibrium uud geometry, keeping the hydrogen-bonded framework frozen, and finding the optimized torsional angles with one angle fixed at zero. All the relevant geometrical data are given in Table I.

The kinetic energy operator for the large amplitude torsional motions was derived in Ref. 12. Even for the rotationless system with total angular momentum $J=0$ for which we performed the calculations presented here, the kinetic energy operator is not simply the sum of one-dimensional kinetic energy operators for rotation about a single fixed axis, but it contains also coupling terms involving the angular momenta generated by the torsional motions. The latter terms include the trimer rotational constants and they are small, but not negligible. ${ }^{13}$ Together with the potential $V\left(\chi_{A}, \chi_{B}, \chi_{C}\right)$, this kinetic energy operator defines the torsional Hamiltonian.

The three-dimensional discrete variable representation (DVR) used to compute the eigenvalues of this Hamiltonian is described in Ref. 13. With an angular grid ranging from $-112.5^{\circ}$ to $+112.5^{\circ}$ for each angle, in steps of $7.5^{\circ}$, the eigenvalues are converged to about $0.01 \mathrm{~cm}^{-1}$. The DVR product basis $\chi_{A}(i) \chi_{B}(j) \chi_{C}(k)$ with $i, j, k=1, \ldots, 31$ was adapted to the irreps of $G_{6}$, which for each irrep reduces the dimension of the basis by about a factor of 6 . For the (vibrationally averaged) trimer rotational constants we took the values $A=B=0.22172 \mathrm{~cm}^{-1}$ and $C=0.11086 \mathrm{~cm}^{-1}$ determined experimentally ${ }^{4}$ for the ground state of $\left(\mathrm{H}_{2} \mathrm{O}\right)_{3}$. For $\left(\mathrm{D}_{2} \mathrm{O}\right)_{3}$ we took the experimental ${ }^{5}$ values $A=B=0.19334 \mathrm{~cm}^{-1}$ and $C=0.10302 \mathrm{~cm}^{-1}$.

\section{RESULTS AND DISCUSSION}

The results computed with different potentials are shown and compared with experiment in Table II and Fig. 2 for $\left(\mathrm{H}_{2} \mathrm{O}\right)_{3}$ and in Table III and Fig. 3 for $\left(\mathrm{D}_{2} \mathrm{O}\right)_{3}$. In order to investigate the effect of the three-body interactions, we performed the calculations with the CC-pol potential at three different levels: (i) with the potential as a sum of pair interactions only, labeled CC-pol(2B); (ii) with the three-body polarization (or induction) energy added that is included in the polarizable CC-pol potential, labeled CC-pol; and (iii) with the full three-body interaction added that includes also the nonadditive exchange interactions, labeled CC-pol $+3 \mathrm{~B}$. The HBB potential is a nonpolarizable pair potential, so we 
TABLE I. Parameters of the equilibrium geometry of $\left(\mathrm{H}_{2} \mathrm{O}\right)_{3}$ computed with different potentials: monomer bond length $r_{\mathrm{OH}}, \mathrm{HOH}$ angle, trimer center of mass distance $R$, angle $\theta$ between monomer torsional axes and center of mass axes, torsional angles $\chi_{A}, \chi_{B}$, and $\chi_{C}$, binding energy $D_{e}$, torsional angles at barrier, and barrier height $\Delta E$. The equibrium geometry was obtained (see text) by minimization on the 12-dimensional intermolecular energy surface for each potential given, with the restriction that the hydrogen-bonded framework has threefold symmetry. The barrier $\Delta E$ was obtained by allowing torsions of the monomers around the axes fixed to this framework, setting $\chi_{B}=0$, and optimizing $\chi_{A}$ and $\chi_{C}$.

\begin{tabular}{lcccccc}
\hline \hline & CC-pol(2B) & CC-pol $^{\mathrm{a}}$ & CC-pol+3B & HBB +3B & TTM2. $^{\mathrm{a}}$ & VRT(ASP-W)III $^{\mathrm{a}}$ \\
\hline$r_{\mathrm{OH}}(\AA)$ & 0.9716 & 0.9716 & 0.9716 & 0.9613 & 0.9572 & 0.9571 \\
$\angle \mathrm{HOH}(\mathrm{deg})$ & 104.66 & 104.66 & 104.66 & 104.20 & 104.52 & 104.52 \\
$R(\AA)$ & 2.878 & 2.836 & 2.796 & 2.827 & 2.815 & 2.830 \\
$\theta(\mathrm{deg})$ & 20.76 & 20.68 & 20.59 & 19.69 & 15.5 & 22.0 \\
$\chi_{A}(\mathrm{deg})$ & 49 & 49 & 48.5 & 49 & 36 & 49 \\
$\chi_{B}(\mathrm{deg})$ & 47 & 47 & 46 & 45 & 42 & 52 \\
$\chi_{C}(\mathrm{deg})$ & -54 & -54 & -53 & -52 & -49 & -54 \\
$D_{e}\left(\mathrm{~cm}^{-1}\right)^{\mathrm{b}}$ & 4915 & 5295 & 5623 & 5174 & 5386 & 5433 \\
$\chi_{A}(\mathrm{deg})$ & 54 & 54 & 53 & 53 & 45 & 53 \\
$\chi_{B}(\mathrm{deg})$ & 0 & 0 & 0 & 0 & 0 & 0 \\
$\chi_{C}(\mathrm{deg})$ & -53 & -53 & -52 & -52 & -47 & -54 \\
$\Delta E\left(\mathrm{~cm}^{-1}\right)^{\mathrm{b}}$ & 92.8 & 100.6 & 99.5 & 94.0 & 21.1 & 261.7 \\
\hline \hline
\end{tabular}

${ }^{\mathrm{a}}$ Includes the three-body induction energy computed with the polarizable potential.

${ }^{b}$ Best estimates from the most recent ab initio calculations (Ref. 51) with all coordinates optimized: $D_{e}$ $=5531 \mathrm{~cm}^{-1}$ and $\Delta E=81 \mathrm{~cm}^{-1}$.

added the same three-body polarization and exchange interactions as for $\mathrm{CC}-$ pol+3B; this potential is then labeled $\mathrm{HBB}+3 \mathrm{~B}$. Actually, we used two versions of the HBB pair potential from Refs. 30 and 31 . The results were very similar, so we just present them for the most recent HBB potential of Ref. 31 which gave the most accurate water dimer VRT levels. The TTM2.1 and VRT(ASP-W)III potentials are polarizable and we have used their polarization components to account for the nonadditive interactions.

Let us first discuss the role of three-body interactions by comparing CC-pol(2B), CC-pol, and CC-pol+3B. In Table I, we see that the binding energy $D_{e}$ is substantially increased by the nonadditive induction terms included in the polarizable CC-pol potential and, again, by the three-body exchange interactions additionally included in CC-pol $+3 \mathrm{~B}$. In total, the three-body interactions contribute about $13 \%$ to the bind- ing energy. Also the torsional barrier $\Delta E$ is increased by the three-body induction, by about $8 \%$, while the three-body exchange terms have only a minor effect. The CC-pol+3B barrier of $99.5 \mathrm{~cm}^{-1}$ is $23 \%$ larger than the best estimate of this barrier amounting to $81 \mathrm{~cm}^{-1} .{ }^{51}$ The uncertainty of the latter value resulting from the use of different basis sets and different types of extrapolation to the complete basis set limit is about $6 \mathrm{~cm}^{-1}$. Since the accuracy of the $a b$ initio calculations leading to the CC-pol+3B potential is very close to that of Ref. 51, it is clear that the differences result from the reduced dimensionality model used by us in the determination of the barrier and possibly also from inaccuracies introduced by the fits. The calculations of Ref. 51 correspond to a 21-dimensional search for the torsional transition state, whereas we searched only in three dimensions. These effects have been investigated in Ref. 45. The 12-dimensional

TABLE II. Torsional energy levels of $\left(\mathrm{H}_{2} \mathrm{O}\right)_{3}$ for $J=0$ from experiment (Ref. 4) and computed with different potentials.

\begin{tabular}{lccccccc}
\hline \hline$G_{6}$ irrep & Expt. & CC-pol(2B) & CC-pol $^{\text {a }}$ & CC-pol+3B & HBB +3B & TTM2.1 $^{\text {a }}$ & VRT(ASP-W)III $^{\text {a }}$ \\
\hline$k=0$ & 0 & 0 & 0 & 0 & 0 & 0 & 0 \\
$k= \pm 1$ & 22.698 & 18.791 & 18.175 & 19.443 & 19.623 & 47.550 & 8.357 \\
$k= \pm 2$ & 65.630 & 54.465 & 53.092 & 56.204 & 56.795 & 109.420 & 24.579 \\
$k=3$ & 87.053 & 73.900 & 72.219 & 75.873 & 75.572 & 108.334 & 32.476 \\
$k=0$ & & 154.062 & 158.980 & 160.471 & 160.535 & 143.022 & 166.503 \\
$k=3$ & & 157.662 & 159.856 & 165.107 & 164.661 & 184.392 & 167.266 \\
$k=3$ & 169.130 & 173.653 & 175.393 & 169.316 & 205.636 & 195.057 \\
$k= \pm 2$ & 166.147 & 168.683 & 173.151 & 170.644 & 188.766 & 201.195 \\
$k= \pm 1$ & & 194.836 & 197.239 & 202.659 & 204.131 & 228.214 & 218.083 \\
$k=0$ & 215.887 & 218.453 & 223.074 & 217.157 & 229.146 & 222.879 \\
$k= \pm 1$ & & 232.855 & 237.388 & 242.795 & 239.593 & 263.473 & 260.564 \\
$k= \pm 2$ & 240.817 & 244.905 & 251.071 & 249.597 & 286.039 & 263.367 \\
$k=3$ & 277.044 & 282.044 & 287.880 & 280.724 & 294.184 & 279.203 \\
$D_{e}-D_{0}$ & 249.5 & 263.5 & 263.3 & 263.0 & 141.9 & 396.5 \\
\hline
\end{tabular}

${ }^{\mathrm{a}}$ Includes the three-body induction energy computed with the polarizable potential. 
TABLE III. Torsional energy levels of $\left(\mathrm{D}_{2} \mathrm{O}\right)_{3}$ for $J=0$ from experiment (Ref. 5) and computed with different potentials.

\begin{tabular}{lccccccc}
\hline \hline$G_{6}$ irrep & Expt. & CC-pol(2B) & CC-pol $^{\mathrm{a}}$ & CC-pol+3B & HBB +3B & TTM2.1 & VRT(ASP-W)III \\
\hline$k=0$ & 0 & 0 & 0 & 0 & 0 & 0 & 0 \\
$k= \pm 1$ & 8.539 & 7.732 & 7.488 & 8.036 & 8.205 & 26.350 & 2.401 \\
$k= \pm 2$ & 27.992 & 24.422 & 23.615 & 25.129 & 25.691 & 64.602 & 7.273 \\
$k=3$ & 41.100 & 34.684 & 33.338 & 35.350 & 36.080 & 81.560 & 9.758 \\
$k=3$ & 90.381 & 99.914 & 103.067 & 106.185 & 105.310 & 120.059 & 153.205 \\
$k= \pm 2$ & 98.099 & 109.343 & 112.041 & 115.102 & 114.013 & 130.742 & 157.289 \\
$k=0$ & & 118.691 & 122.643 & 123.489 & 125.551 & 94.074 & 165.951 \\
$k= \pm 1$ & & 125.304 & 127.754 & 130.837 & 131.816 & 147.033 & 166.021 \\
$k=3$ & 141.481 & 145.858 & 145.676 & 139.223 & 126.457 & 169.522 \\
$k=0$ & 155.910 & 158.634 & 160.425 & 153.972 & 175.783 & 174.070 \\
$k= \pm 1$ & & 163.114 & 167.614 & 170.568 & 168.471 & 178.574 & 199.736 \\
$k= \pm 2$ & 167.444 & 171.751 & 174.602 & 171.517 & 182.751 & 200.949 \\
$k=0$ & 192.338 & 195.826 & 201.827 & 201.007 & 220.304 & 210.713 \\
$k= \pm 2$ & 191.634 & 196.304 & 201.411 & 203.810 & 214.061 & 221.824 \\
$k=3$ & 195.355 & 199.451 & 203.371 & 200.907 & 203.604 & 209.630 \\
$k= \pm 1$ & 198.889 & 202.223 & 207.822 & 206.279 & 236.795 & 217.260 \\
$k=0$ & 223.287 & 226.944 & 228.489 & 218.384 & 239.769 & 211.219 \\
$D_{e}-D_{0}$ & 203.3 & 212.9 & 214.3 & 213.1 & 110.9 & 312.4 \\
\hline \hline
\end{tabular}

${ }^{\mathrm{a} I n c l u d e s}$ the three-body induction energy computed with the polarizable potential.

search in that work on the SAPT- $5 \mathrm{~s}+3 \mathrm{~B}$ potential energy surface resulted in a barrier of $52 \mathrm{~cm}^{-1}$. This barrier increased to $72 \mathrm{~cm}^{-1}$ after the nonadditive energies at the characteristic trimer configurations obtained in the potential search were recalculated $a b$ initio. The $20 \mathrm{~cm}^{-1}$ difference indicated the fitting errors in the three-body potential of Ref. 45. The overall fitting error in this potential was $0.07 \mathrm{kcal} / \mathrm{mol}\left(24 \mathrm{~cm}^{-1}\right),{ }^{45}$ so that discrepancies of this size are to be expected. The authors of Ref. 45 performed also a three-dimensional optimization, analogous to that done by us, and obtained a barrier of $66 \mathrm{~cm}^{-1}$ which increased to $89 \mathrm{~cm}^{-1}$ when the nonadditive energies were recalculated $a b$ initio. Thus, the reduced dimensionality model used in our work tends to increase the barrier by about 14 to $17 \mathrm{~cm}^{-1}$. So we may expect that a 12-dimensional optimization of the $\mathrm{CC}-$ pol $+3 \mathrm{~B}$ potential would result in a barrier of about

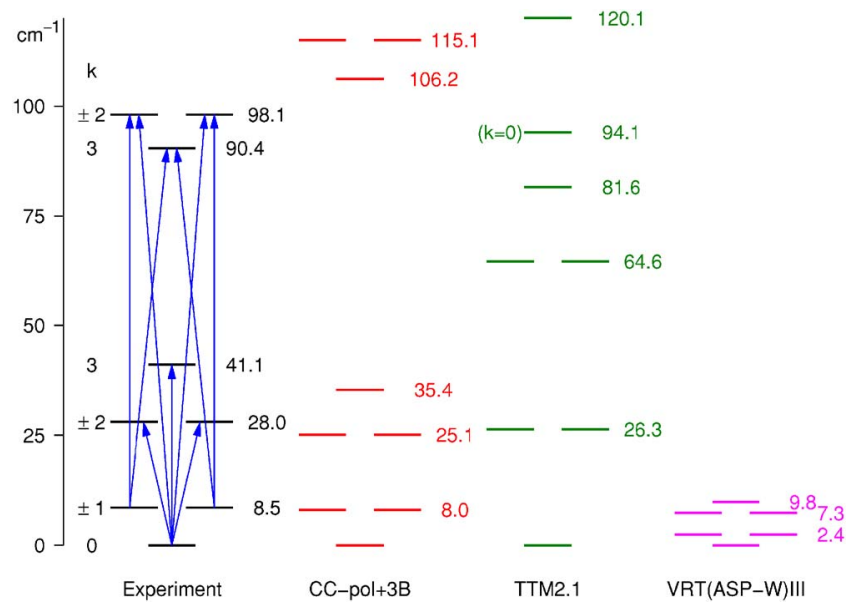

FIG. 3. (Color online) Torsional levels (in $\left.\mathrm{cm}^{-1}\right)$ of $\left(\mathrm{D}_{2} \mathrm{O}\right)_{3}$ from experiment (Ref. 5) and from calculations with different potentials. Arrows indicate the observed transitions. The levels from the $\mathrm{HBB}+3 \mathrm{~B}$ potential are very similar to the CC-pol+3B levels.
$85 \mathrm{~cm}^{-1}$, fairly close to the best estimate of Ref. 51 . The use of $a b$ initio nonadditive contributions would probably deteriorate this agreement, but several other sources of uncertainty would have to be taken into account at this level of accuracy (in particular, inaccuracies of the two-body potential and monomer-flexibility effects) to make meaningful comparisons. We should also point out that the rather small contribution of the nonadditive effects to the barrier height $\left(6.7 \mathrm{~cm}^{-1}\right.$ or $7.2 \%$ increase) is probably an underestimate resulting from inaccuracies in the fit of the nonadditive energies. In the 12-dimensional optimizations of Ref. 45, these effects increased the barrier from 48 to $52 \mathrm{~cm}^{-1}$ only, but the latter value became equal to $72 \mathrm{~cm}^{-1}$ when the nonadditive contribution was recalculated $a b$ initio.

Table I compares also the depth of the potentials at the minimum. The best estimate for $D_{e}$ of Ref. 51 is $5531 \mathrm{~cm}^{-1}$ with an error estimate of $23 \mathrm{~cm}^{-1}$, whereas the best estimate of Ref. 45 is $5631 \pm 70 \mathrm{~cm}^{-1}$. Thus, the two estimates are consistent to within the joint error bars, a very reasonable agreement taking into account that the two values differ in the inclusion of monomer relaxation effects. The CC-pol+3B surface has a well depth $D_{e}$ of $5623 \mathrm{~cm}^{-1}$, in excellent agreement with the estimate of Ref. 45. Our definition of $D_{e}$ is consistent with that of Ref. 45, but one should point out that our $D_{e}$ was obtained from restricted dimensionality (five-dimensional) optimizations and would be increased in a full 12-dimensional search. The value of $D_{e}$ from the $\mathrm{HBB}+3 \mathrm{~B}$ potential is too small, which is due to the fact that the HBB pair potential is too shallow. ${ }^{28}$

In Tables II and III, we see that the torsional frequencies of both $\left(\mathrm{H}_{2} \mathrm{O}\right)_{3}$ and $\left(\mathrm{D}_{2} \mathrm{O}\right)_{3}$ do not change much by the inclusion of the three-body induction terms: the splittings decrease for $\left(\mathrm{H}_{2} \mathrm{O}\right)_{3}$ by $2 \%-3 \%$. The frequencies are increased by $2 \%-3 \%$ compared to the $\mathrm{CC}-\operatorname{pol}(2 \mathrm{~B})$ values when the complete three-body potential is used, including exchange. 
Thus, the overall effect of the exchange nonadditive contributions is fairly significant. Since the barrier heights of CC-pol and CC-pol+3B are nearly the same, clearly, it is not only the barrier height that is important for the torsional frequencies. The inclusion of the exchange nonadditive contributions significantly improves the agreement with the measured frequencies compared to the case when only the polarization nonadditive components are used. One should notice that there are two effects of the three-body interactions: a direct one, due to their contribution to the torsional potential, and an indirect one, since these interactions make the equilibrium geometry of the water trimer more compact which changes the contribution of the pair potential.

Before we compare the frequencies obtained from different potentials, let us remind the reader that the splittings between the lowest four levels (or six, if we take into account that the $k= \pm 1$ and $k= \pm 2$ levels are doubly degenerate) are due to the $u$ - $d$ tunneling flips. Indeed, we observe for both $\left(\mathrm{H}_{2} \mathrm{O}\right)_{3}$ and $\left(\mathrm{D}_{2} \mathrm{O}\right)_{3}$ that the splitting of the ground state tunneling levels nicely corresponds to the simple theoretical model that predicts a picture similar to the $\pi$ molecular orbital diagram of benzene, ${ }^{12,22}$ see Figs. 2 and 3. The higher levels correspond to excitation of the torsional vibration mode. Each of the vibrational (fundamental and higher) excited states is split by tunneling again. It should be noted that the wave function of the fundamental mode has a node at the equilibrium geometry, so that the energy order of the $G_{6}$ symmetry-adapted linear combinations for different irreps will be different from the ground state order. This picture is most clearly observed for $\left(\mathrm{D}_{2} \mathrm{O}\right)_{3}$ since the tunneling splittings are about half of those for $\left(\mathrm{H}_{2} \mathrm{O}\right)_{3}$, due to the two times larger torsional moment of inertia of the monomers. We observe that the energy order of the lowest excited states with $k=3$ and $k= \pm 2$ in $\left(\mathrm{D}_{2} \mathrm{O}\right)_{3}$ is reversed with respect to the order of the ground state (where $k= \pm 2$ and $k=3$ are the highest tunneling levels). For $\left(\mathrm{H}_{2} \mathrm{O}\right)_{3}$, the highest tunneling level of the ground state has an energy close to the torsional barrier, so that tunneling and vibrational excitation are mixed in the levels above and the picture becomes more complicated. The same complication arises for the higher levels of $\left(\mathrm{D}_{2} \mathrm{O}\right)_{3}$.

The levels computed from the CC-pol+3B and $\mathrm{HBB}+3 \mathrm{~B}$ potentials agree well with experiment, both for $\left(\mathrm{H}_{2} \mathrm{O}\right)_{3}$ and $\left(\mathrm{D}_{2} \mathrm{O}\right)_{3}$. The torsional tunneling splittings are slightly too small for both potentials. Since the values from the two potentials agree so well with each other, the main reason for this deviation from experiment is probably the inaccuracy of the restricted dimensionality model used to describe the torsional motions. Indeed, as discussed above, in such a model the barriers are overestimated, which reduces the splittings. The torsional excited states observed in $\left(\mathrm{D}_{2} \mathrm{O}\right)_{3}$ are predicted about $17 \%$ too high by both potentials, which is not surprising since it might be expected that the restricted dimensionality model breaks down for higher energies. It might be mentioned here that both the CC-pol and HBB potentials have been shown ${ }^{27,29,31}$ to produce tunneling splittings, intermolecular vibrational frequencies, and rotational constants for the $\mathrm{H}_{2} \mathrm{O}$ and $\mathrm{D}_{2} \mathrm{O}$ dimers in very good agreement with experiment.
The trimer levels computed in Ref. 6 applying the SAPT-5s water pair potential and three-body nonadditive energies calculated $a b$ initio at the trimer grid points using the Hartree-Fock (HF) level of theory remain the most accurate theoretical predictions to date. The errors of these levels for the $\mathrm{H}_{2} \mathrm{O}$ trimer range from $3 \%$ to $11 \%$, whereas $\mathrm{CC}$-pol $+3 \mathrm{~B}$ gives errors between $13 \%$ and $14 \%$. For the $\mathrm{D}_{2} \mathrm{O}$ trimer, some levels computed from the CC-pol $+3 \mathrm{~B}$ potential are closer to experiment than those from SAPT- $5 \mathrm{~s}+3 \mathrm{~B}(\mathrm{HF})$, but overall the latter are in better agreement. This behavior was unexpected considering that the dimer spectra predicted by the CC-pol potential are significantly more acurate ${ }^{27,29}$ than those predicted by SAPT-5s. The reason for the greater accuracy of the trimer levels computed from SAPT- $5 \mathrm{~s}+3 \mathrm{~B}(\mathrm{HF})$ is probably a fortuitous cancellation of errors. Table IV in Ref. 45 shows that the upd tunneling barrier on SAPT-5s at the two-body level from a 12-dimensional optimization is $48 \mathrm{~cm}^{-1}$. Thus, if the true nonadditive contribution to the barrier is $25 \mathrm{~cm}^{-1}$, as discussed above, this would give a barrier of $73 \mathrm{~cm}^{-1}$, slightly too low. However, the actual barrier from a threedimensional optimization is $89 \mathrm{~cm}^{-1}$. ${ }^{45}$ Thus, in the case of SAPT-5s, the error due to the reduced dimensionality model is partly compensated by the underestimation of the barrier on the 12-dimensional surface. By contrast, the 12-dimensional CC-pol $+3 \mathrm{~B}$ potential probably gives an overestimate of the barrier, which is reinforced by the reduced dimensionality effect. On the other hand, the 3B fit of Ref. 45 tends to underestimate the nonadditive contribution, and probably this inaccuracy improves the predictions.

The $a b$ initio TTM2.1 potential yields tunneling splittings for the water trimer that are much too large. In Table I we can see that this is because the torsional barrier $\Delta E$ is about four times too small. This picture is similar to that observed $^{53,56}$ for the empirical TIP4P potential, very popular in simulations of liquid water. The very low barrier explains also why the torsional zero-point energy $D_{e}-D_{0}$ from the TTM2.1 potential is much smaller than for the other potentials. Moreover, the equilibrium geometry of the water trimer predicted by this potential differs significantly from the geometries obtained with the other potentials. Another consequence of such a small barrier is that the experimentally confirmed typical pattern of the tunneling splittings is not reproduced by the TTM2.1 potential. Perhaps this is not surprising, as this potential also yielded tunneling splittings and intermolecular vibrational frequencies that do not agree with experiment for the water dimer. ${ }^{27,29,31}$ The VRT(ASP-W)III potential that was fitted to these frequencies for $\left(\mathrm{D}_{2} \mathrm{O}\right)_{2}$ yields tunneling splittings for $\left(\mathrm{H}_{2} \mathrm{O}\right)_{3}$ and $\left(\mathrm{D}_{2} \mathrm{O}\right)_{3}$ that are much too small. The torsional zero-point energy is much higher than for the other potentials. By looking at Table I again, we observe that these discrepancies are a consequence of the torsional barrier $\Delta E$ being too high by a factor of 3 . More or less the same defects have been observed ${ }^{53,56}$ for the ASP-W potentials ${ }^{57}$ from which the VRT(ASP-W) potentials were derived. Thus, it seems that the water trimer spectrum probes the potential for different geometries than the water dimer spectrum to which the VRT(ASP-W) potentials were fitted. 


\section{CONCLUSIONS}

In this paper, we have tested several recent water potentials obtained directly from $a b$ initio calculations or through a fit of the water dimer high-resolution terahertz spectrum. This was done by computing the torsional tunneling splittings and vibrational frequencies of the water trimer and by comparing the results with the experimental data available for both $\left(\mathrm{H}_{2} \mathrm{O}\right)_{3}$ and $\left(\mathrm{D}_{2} \mathrm{O}\right)_{3}$. The torsional energy levels were calculated in a restricted dimensionality model based on the fact that the torsional frequencies, in particular those that correspond to a low barrier tunneling motion, are considerably lower than those of the vibrational modes of the strong hydrogen-bonded "framework" of the water trimer. We investigated also the role of nonadditive three-body interactions, not tested by the water dimer spectrum to which the same potentials were recently applied.

We have found that the polarizable CC-pol potential of Refs. 27-29 and the nonpolarizable HBB potential of Refs. 30 and 31, supplemented with the three-body nonadditive potential of Ref. 45, gave good agreement with the measured torsional frequencies for both $\left(\mathrm{H}_{2} \mathrm{O}\right)_{3}$ and $\left(\mathrm{D}_{2} \mathrm{O}\right)_{3}$. This is gratifying since the CC-pol and $\mathrm{HBB}$ potentials gave also very good agreement with the experimental high-resolution spectra of the $\mathrm{H}_{2} \mathrm{O}$ and $\mathrm{D}_{2} \mathrm{O}$ dimers. Thus, one may conclude that $a b$ initio calculations have now reached such an accuracy that reliable and useful intermolecular force fields can be obtained from first principles. Such force fields can be applied to predict the properties of small water clusters, as well as of liquid water and ice. The CC-pol potential has already been used ${ }^{29}$ in MD simulations of liquid water and indeed it reproduced a number of the properties of water with an accuracy that is about as good as for the best empirical potentials fitted to these properties. The three-body interactions in the water trimer increase significantly the binding energy $D_{e}$ and, consequently, the dissociation energy $D_{0}$. Their effect on the torsional energy barrier and tunneling frequencies is somewhat less significant, although this significance may increase when the dimensionality restrictions of the torsional model are removed and the quality of the potential fits is improved. The frequencies from the $\mathrm{CC}$-pol $+3 \mathrm{~B}$ and $\mathrm{HBB}+3 \mathrm{~B}$ potentials are very similar and the remaining small deviations from the experimental frequencies are probably due to the shortcomings of the restricted dimensionality model and inaccuracies of the fits. Another ab initio potential, the TTM2.1 potential of Ref. 26, yields a torsional energy barrier in the water trimer that is about four times too small and tunneling frequencies that are much too high. Also the tunneling splittings and intermolecular vibrational frequencies of the water dimer from this potential were unrealistic. $^{27,29,31}$ The semiempirical VRT(ASP-W)III potential fitted to the water dimer spectrum yields a torsional barrier for the trimer that is about three times too high and tunneling splittings that are much smaller than the measured values. Hence, one may also conclude that the water trimer torsional spectrum senses the water potential for geometries not probed by the water dimer spectrum.

\section{ACKNOWLEDGMENTS}

We thank Dr. Sotiris Xantheas for making available the code of the TTM2.1 potential, Dr. Nir Goldman for the VRT(ASP-W)III potential code, and Professor Joel Bowman and Dr. Xinchuan Huang for the HBB potential code. We also acknowledge useful discussions with Dr. Gerrit C. Groenenboom. This work was partly supported by NSF under Grant No. CHE-0555979.

${ }^{1}$ N. Pugliano and R. J. Saykally, Science 257, 1937 (1992).

${ }^{2}$ K. Liu, J. G. Loeser, M. J. Elrod, B. C. Host, J. A. Rzepiela, and R. J. Saykally, J. Am. Chem. Soc. 116, 3507 (1994).

${ }^{3}$ K. Liu, M. J. Elrod, J. G. Loeser, J. D. Cruzan, N. Pugliano, M. Brown, J. Rzepiela, and R. J. Saykally, Faraday Discuss. Chem. Soc. 97, 35 (1994).

${ }^{4}$ M. G. Brown, M. R. Viant, R. P. McLaughlin, C. J. Keoshian, E. Michael, J. D. Cruzan, R. J. Saykally, and A. van der Avoird, J. Chem. Phys. 111, 7789 (1999)

${ }^{5}$ M. R. Viant, M. G. Brown, J. D. Cruzan, R. J. Saykally, M. Geleijns, and A. van der Avoird, J. Chem. Phys. 110, 4369 (1999).

${ }^{6}$ G. C. Groenenboom, E. M. Mas, R. Bukowski, K. Szalewicz, P. E. S. Wormer, and A. van der Avoird, Phys. Rev. Lett. 84, 4072 (2000).

${ }^{7}$ Z. Bačić, Comput. Phys. Commun. 145, 184 (2002).

${ }^{8}$ D. Sabo, Z. Bačić, S. Graf, and S. Leutwyler, J. Chem. Phys. 110, 5745 (1999).

${ }^{9}$ D. Sabo, Z. Bačić, S. Graf, and S. Leutwyler, J. Chem. Phys. 111, 5331 (1999).

${ }^{10}$ D. Sabo, Z. Bačić, S. Graf, and S. Leutwyler, J. Chem. Phys. 111, 10727 (1999).

${ }^{11}$ M. Geleijns and A. van der Avoird, J. Chem. Phys. 110, 823 (1999).

${ }^{12}$ A. van der Avoird, E. H. T. Olthof, and P. E. S. Wormer, J. Chem. Phys. 105, 8034 (1996)

${ }^{13}$ E. H. T. Olthof, A. van der Avoird, P. E. S. Wormer, K. Liu, and R. J. Saykally, J. Chem. Phys. 105, 8051 (1996).

${ }^{14}$ J. M. Sorenson, J. K. Gregory, and D. C. Clary, Chem. Phys. Lett. 263, 680 (1996).

${ }^{15}$ D. Sabo, Z. Bačić, S. Graf, and S. Leutwyler, Chem. Phys. Lett. 261, 318 (1996).

${ }^{16}$ T. Bürgi, S. Graf, S. Leutwyler, and W. Klopper, J. Chem. Phys. 103, 1077 (1995)

${ }^{17}$ W. Klopper, M. Schütz, H.-P. Lüthi, and S. Leutwyler, J. Chem. Phys. 103, 1085 (1995)

${ }^{18}$ W. Klopper and M. Schütz, Chem. Phys. Lett. 237, 536 (1995).

${ }^{19}$ D. Sabo, Z. Bačić, T. Bürgi, and S. Leutwyler, Chem. Phys. Lett. 244, 283 (1995).

${ }^{20}$ J. G. C. M. van Duijneveldt-van de Rijdt and F. B. van Duijneveldt, Chem. Phys. Lett. 237, 560 (1995).

${ }^{21}$ J. K. Gregory and D. C. Clary, J. Chem. Phys. 103, 8924 (1995).

${ }^{22}$ D. J. Wales, J. Am. Chem. Soc. 115, 11180 (1993).

${ }^{23}$ M. Schütz, T. Bürgi, S. Leutwyler, and H. B. Bürgi, J. Chem. Phys. 99, 5228 (1993).

${ }^{24}$ M. Schütz, T. Bürgi, S. Leutwyler, and H. B. Bürgi, J. Chem. Phys. 100, 1780 (1994)

${ }^{25}$ P. E. S. Wormer and A. van der Avoird, Chem. Rev. (Washington, D.C.) 100, 4109 (2000)

${ }^{26}$ G. S. Fanourgakis and S. S. Xantheas, J. Phys. Chem. A 110, 4100 (2006).

${ }^{27}$ R. Bukowski, K. Szalewicz, G. C. Groenenboom, and A. van der Avoird, Science 315, 1249 (2007).

${ }^{28}$ R. Bukowski, K. Szalewicz, G. C. Groenenboom, and A. van der Avoird, "Polarizable interaction potential for water from coupled cluster calculations. I. Analysis of dimer potential energy surface," J. Chem. Phys. (in press).

${ }^{29}$ R. Bukowski, K. Szalewicz, G. C. Groenenboom, and A. van der Avoird, "Polarizable interaction potential for water from coupled cluster calculations. II. Applications to dimer spectra, virial coefficients, and simulations of liquid water," J. Chem. Phys. (in press).

${ }^{30}$ X. Huang, B. J. Braams, and J. M. Bowman, J. Phys. Chem. A 110, 445 (2006). 
${ }^{31}$ X. Huang, B. J. Braams, J. M. Bowman, R. E. A. Kelly, J. Tennyson, G. C. Groenenboom, and A. van der Avoird, "New ab initio potential energy surface and the vibration-rotation-tunneling levels of $\left(\mathrm{H}_{2} \mathrm{O}\right)_{2}$ and $\left(\mathrm{D}_{2} \mathrm{O}\right)_{2}$, , J. Chem. Phys. (in press).

${ }^{32}$ E. Zwart, J. J. ter Meulen, and W. L. Meerts, Chem. Phys. Lett. 173, 115 (1990).

${ }^{33}$ E. Zwart, J. J. ter Meulen, W. L. Meerts, and L. H. Coudert, J. Mol. Spectrosc. 147, 27 (1991).

${ }^{34}$ G. T. Fraser, Int. Rev. Phys. Chem. 10, 189 (1991).

${ }^{35}$ E. N. Karyakin, G. T. Fraser, and R. D. Suenram, Mol. Phys. 78, 1179 (1993).

${ }^{36}$ J. B. Paul, R. A. Provencal, and R. J. Saykally, J. Phys. Chem. A 102, 3279 (1998)

${ }^{37}$ L. B. Braly, J. D. Cruzan, K. Liu, R. S. Fellers, and R. J. Saykally, J. Chem. Phys. 112, 10293 (2000).

${ }^{38}$ L. B. Braly, K. Liu, M. G. Brown, F. N. Keutsch, R. S. Fellers, and R. J. Saykally, J. Chem. Phys. 112, 10314 (2000).

${ }^{39}$ F. N. Keutsch, N. Goldman, H. A. Harker, C. Leforestier, and R. J. Saykally, Mol. Phys. 101, 3477 (2003).

${ }^{40}$ F. N. Keutsch, L. B. Braly, M. G. Brown, H. A. Harker, P. B. Petersen, C. Leforestier, and R. J. Saykally, J. Chem. Phys. 119, 8927 (2003).

${ }^{41}$ R. S. Fellers, L. B. Braly, R. J. Saykally, and C. Leforestier, J. Chem. Phys. 110, 6306 (1999).

${ }^{42}$ N. Goldman, R. Fellers, M. Brown, L. Braly, C. Keoshian, C. Leforestier, and R. Saykally, J. Chem. Phys. 116, 10148 (2002).
${ }^{43}$ C. Leforestier, F. Gatti, R. Fellers, and R. Saykally, J. Chem. Phys. 117, $8710(2002)$

${ }^{44}$ E. M. Mas, R. Bukowski, K. Szalewicz, G. C. Groenenboom, P. E. S. Wormer, and A. van der Avoird, J. Chem. Phys. 113, 6687 (2000).

${ }^{45}$ E. M. Mas, R. Bukowski, and K. Szalewicz, J. Chem. Phys. 118, 4386 (2003).

${ }^{46}$ J. E. Fowler and H. F. Schaefer, J. Am. Chem. Soc. 117, 446 (1995).

${ }^{47}$ S. S. Xantheas and T. H. Dunning, J. Chem. Phys. 98, 8037 (1993).

${ }^{48}$ S. S. Xantheas and T. H. Dunning, J. Chem. Phys. 99, 8774 (1993).

${ }^{49}$ G. Chałasiński, M. M. Szczęśniak, P. Cieplak, and S. Scheiner, J. Chem. Phys. 94, 2873 (1991).

${ }^{50}$ T. Taketsugu and D. J. Wales, Mol. Phys. 100, 2793 (2002).

${ }^{51}$ J. A. Anderson, K. Crager, L. Fedoroff, and G. S. Tschumper, J. Chem. Phys. 121, 11023 (2004)

${ }^{52}$ M. Takahashi, Y. Watanabe, T. Taketsugu, and D. J. Wales, J. Chem. Phys. 123, 044302 (2005).

${ }^{53}$ T. R. Walsh and D. J. Wales, J. Chem. Soc., Faraday Trans. 92, 2505 (1996).

${ }^{54}$ F. N. Keutsch, R. J. Saykally, and D. J. Wales, J. Chem. Phys. 117, 8823 (2002).

${ }^{55}$ A. Milet, R. Moszynski, P. E. S. Wormer, and A. van der Avoird, J. Phys. Chem. A 103, 6811 (1999).

${ }^{56}$ D. J. Wales, in Advances in Molecular Vibrations and Collision Dynamics, edited by J. M. Bowman and Z. Bačić (JAI, Stamford, 1998), p. 365.

${ }^{57}$ C. Millot and A. J. Stone, Mol. Phys. 77, 439 (1992). 